Di Rienzo, Luca; Haueisen, Jens:

\title{
Numerical comparison of sensor arrays for magnetostatic linear inverse problems based on a projection method
}

URN: $\quad$ urn:nbn:de:gbv:ilm1-2014210127

Published OpenAccess: September 2014

Original published in:

Compel : international journal of computation \& mathematics in electrical \& electronic engineering. - Bradford : Emerald (ISSN-p 0332-1649). - 26 (2007) 2, S. 356-367.

DOI: $\quad 10.1108 / 03321640710727719$

URL: $\quad$ http://dx.doi.org/10.1108/03321640710727719

[Visited: 2014-09-02]

„Im Rahmen der hochschulweiten Open-Access-Strategie für die Zweitveröffentlichung identifiziert durch die Universitätsbibliothek Ilmenau."

"Within the academic Open Access Strategy identified for deposition by Ilmenau University Library."

"Dieser Beitrag ist mit Zustimmung des Rechteinhabers aufgrund einer (DFG-geförderten) Allianz- bzw. Nationallizenz frei zugänglich."

"This publication is with permission of the rights owner freely accessible due to an Alliance licence and a national licence (funded by the DFG, German Research Foundation) respectively."

\section{DFG}

Nationallizenzen 


\section{COMPEL - The international journal for computation and ma in electrical and electronic engineering}

Numerical comparison of sensor arrays for magnetostatic linear inverse problems based on a projection method

Luca Di Rienzo Jens Haueisen

\section{Article information:}

To cite this document:

Luca Di Rienzo Jens Haueisen, (2007),"Numerical comparison of sensor arrays for magnetostatic linear inverse problems based on a projection method", COMPEL - The international journal for computation and mathematics in electrical and electronic engineering, Vol. 26 Iss 2 pp. 356 - 367

Permanent link to this document:

http://dx.doi.org/10.1108/03321640710727719

Downloaded on: 02 September 2014, At: 07:54 (PT)

References: this document contains references to 15 other documents.

To copy this document: permissions@emeraldinsight.com

The fulltext of this document has been downloaded 161 times since 2007*

\section{Users who downloaded this article also downloaded:}

Luca Di Rienzo, Jens Haueisen, Cesare Mario Arturi, (2005),"Three component magnetic field data: Impact on minimum norm solutions in a biomedical application", COMPEL - The international journal for computation and mathematics in electrical and electronic engineering, Vol. 24 Iss 3 pp. 869-881

A. Yao, M. Soleimani, (2012),"A pressure mapping imaging device based on electrical impedance tomography of conductive fabrics", Sensor Review, Vol. 32 Iss 4 pp. 310-317

O. Korostynska, A. Mason, A. Al-Shamma'a, (2014),"Microwave sensors for the non-invasive monitoring of industrial and medical applications", Sensor Review, Vol. 34 Iss 2 pp. 182-191 http://dx.doi.org/10.1108/ SR-11-2012-725

Access to this document was granted through an Emerald subscription provided by 514728 []

\section{For Authors}

If you would like to write for this, or any other Emerald publication, then please use our Emerald for Authors service information about how to choose which publication to write for and submission guidelines are available for all. Please visit www.emeraldinsight.com/authors for more information.

\section{About Emerald www.emeraldinsight.com}

Emerald is a global publisher linking research and practice to the benefit of society. The company manages a portfolio of more than 290 journals and over 2,350 books and book series volumes, as well as providing an extensive range of online products and additional customer resources and services.

Emerald is both COUNTER 4 and TRANSFER compliant. The organization is a partner of the Committee on Publication Ethics (COPE) and also works with Portico and the LOCKSS initiative for digital archive preservation. 
COMPEL

26,2

356
Numerical comparison of sensor arrays for magnetostatic linear inverse problems based on a projection method

\author{
Luca Di Rienzo
}

Politecnico di Milano, Dipartimento di Elettrotecnica, Milano, Italy, and

Jens Haueisen

Institute of Biomedical Engineering and Informatics, Technical University Ilmenau, Ilmenau, Germany

\begin{abstract}
Purpose - To define a methodology for comparing sensor arrays for solving magnetostatic linear inverse problems.

Design/methodology/approach - A singular value decomposition related projection method is used for comparing sensor arrays and we applied it to a biomagnetic inverse problem, as an example. Furthermore, a theoretical reference sensor system is introduced and used as a benchmark for the analysed sensor arrays.

Findings - The method has turned out to be effective in comparing three different theoretical sensor arrays, showing the superiority of the two arrays constituted by three-axial sensors.

Research limitations/implications - The method has been applied only to the case of over-determined problems. The underdetermined case will be considered in future work.

Practical implications - From the applicative point of view, the illustrated methodology is useful when one has to choose between existing sensor arrays or in the design phase of a new sensor array. Originality/value - A new methodology is proposed for comparing sensor arrays. The advantage of the methodology are to take into account the regularization in the solution of the inverse problem and to be general, not depending on a particular source configuration.
\end{abstract}

Keywords Sensors, Magnetic fields

Paper type Research paper

\section{Introduction}

There are many applications in magneto fluid dynamics (Ziolkowski et al., 2006), superconductivity (Bruzzone et al., 2002; Bellina et al., 2002), non-destructive testing (Haueisen et al., 2002), magnetic fields characterization (Scorretti et al., 2004; Rouve et al., 2006), reconstruction of a magnetization distribution (Chadebec et al., 2002), biomagnetism (Arturi et al., 2004; Di Rienzo et al., 2005) that can be formulated as magnetostatic linear inverse problems (IPs).

In their direct formulation this kind of problems are typically described by the following equation:

$$
\mathbf{B}=L \cdot \mathbf{p}_{\text {true }}+\boldsymbol{\varepsilon}
$$

Electrical and Electronic Engineering

Vol. 26 No. 2, 2007

pp. $356-367$

(c) Emerald Group Publishing Limited 0332-1649

DOI 10.1108/03321640710727719 the kernel matrix, $\mathbf{p}_{\text {true }}$ is the source distribution represented by a column vector of where $\mathbf{B}$ is the column vector of $m$ scalar magnetic field measurements, $L \in \Re^{m \times n}$ is 
length $n$ and $\boldsymbol{\varepsilon}$ is the column vector representing the noise. In IPs a relevant source of uncertainty can be due to system inaccuracies (e.g. sensor alignments or sensor position variations). Those inaccuracies affect the kernel matrix $L$ and are not taken into account in the error analysis proposed here. On the other hand, they can be neglected to some extent in biomagnetic applications like the one analyzed in our work.

When these problems are over-determined (i.e. the number of measurements $m$ is higher than the number of unknown parameters $n$ ) and ill-posed, the solution is typically obtained with the help of the truncated singular value decomposition (TSVD) as a regularization scheme (Vogel, 2002; Hansen, 1998; Golub and Van Loan, 1989; Shim and Cho, 1981; Nalbach and Dössel, 2002; Kemppainem and Ilmoniemi, 1989).

After computing the singular value decomposition (SVD) of $L: L=U \Sigma V^{\mathrm{T}}$, where the matrices $U=\left(\boldsymbol{u}_{1}, \boldsymbol{u}_{2}, \ldots, \boldsymbol{u}_{n}\right) \in \mathfrak{R}^{m \times m}$ and $V=\left(\boldsymbol{v}_{1}, \boldsymbol{v}_{2}, \ldots, \boldsymbol{v}_{n}\right) \in \mathfrak{R}^{n \times n}$ are with orthonormal columns and where $\Sigma=\operatorname{diag}\left(\sigma_{1}, \sigma_{2}, \ldots, \sigma_{g}\right), g=\operatorname{rank}(L)$ has non-negative diagonal elements (singular values), inverting the linear system (1) the following solution is obtained:

$$
\begin{aligned}
L^{-1} \mathbf{B} & =V \Sigma^{-1} U^{\mathrm{T}} \mathbf{B}=\sum_{i=1}^{g} \frac{\boldsymbol{u}_{i}^{\mathrm{T}} \cdot \mathbf{B}}{\sigma_{i}} \mathbf{v}_{i}=V \Sigma^{-1} U^{\mathrm{T}} L \mathbf{p}_{\text {true }}+V \Sigma^{-1} U^{\mathrm{T}} \boldsymbol{\varepsilon} \\
& =\mathbf{p}_{\text {true }}+\sum_{i=1}^{g} \frac{\mathbf{u}_{i}^{\mathrm{T}} \cdot \boldsymbol{\varepsilon}}{\sigma_{i}} \mathbf{v}_{i}
\end{aligned}
$$

The small singular values at denominator in equation (2) cause instability. In order to avoid this instability, only the first $r$ terms corresponding to the first $r$ singular values are kept, obtaining the TSVD regularized solution:

$$
\mathbf{p}_{\mathrm{TSVD}}=\sum_{i=1}^{r} \frac{\mathbf{u}_{i}^{\mathrm{T}} \cdot \mathbf{B}}{\sigma_{i}} \mathbf{v}_{i}
$$

The proper choice of the order $r$ of the TSVD depends on the noise level in the data and can be performed according to different criteria (Vogel, 2002; Hansen, 1998; Shim and Cho, 1981).

The solution given by equation (3) is the minimum norm solution (MNS) of the linear system:

$$
\mathbf{B}=L^{r} \cdot \mathbf{p}
$$

where the matrix $L$ is replaced by a truncated version $L^{r}$ of rank $r$ given by the following expansion:

$$
L^{r}=\sum_{i=1}^{r} \mathbf{u}_{i} \sigma_{i} \mathbf{v}_{i}^{\mathrm{T}}
$$

Often one has to choose between different geometries of magnetic sensor arrays. For this scope, it is important to introduce theoretical criteria to compare sensor arrays performances.

A typical cost function used in sensor array optimization is given by the condition number $(\mathrm{CN})$ of the kernel matrix (Bruzzone et al., 2002; Bellina et al., 2002; Rouve et al., 2006; Arturi et al., 2004; Di Rienzo et al., 2005). The analysis based on the CN is limited by the fact that the solution of an ill-posed IP is obtained by a regularization

\section{Numerical comparison of sensor arrays}

357 
COMPEL

26,2

358 algorithm and the $\mathrm{CN}$ of the regularization algorithm is different from the $\mathrm{CN}$ of the kernel matrix.

Furthermore, while the commonly applied $\mathrm{CN}$ analysis is based, by definition, only on the first and the last singular values, the projection analysis used here is based on all the right singular vectors and hence is expected to give a more comprehensive insight.

The principle of the projection method used in our paper was introduced in Nalbach and Dössel (2002). We expand the basic principle to regularized TSVD problems. Furthermore, we introduce a theoretical measurement system which completely samples the magnetic field and works as a gold standard reference. We then apply the comparison method to kernel matrices representing theoretical sensor setups used in Magnetocardiography and described in Arturi et al. (2004) and in Di Rienzo et al. (2005).

\section{Comparison criteria based on TSVD projections}

In order to compare two different sensor arrays geometries, that can be made of different numbers of sensors, let us consider the associated kernel matrices $L_{1} \in \mathfrak{R}^{m_{1} \times n}$ and $L_{2} \in \mathfrak{R}^{m_{2} \times n}$. After applying TSVD with truncation order $r_{1}$ to $L_{1}$ and with truncation order $r_{2}$ to $L_{2}$, the matrices $L_{1}$ and $L_{2}$ are replaced by their truncated versions $L_{1}^{r 1}$ and $L_{2}^{r 2}$, respectively. After computing SVD:

$$
L_{1}=U_{1} \Sigma_{1} V_{1}^{\mathrm{T}}, \quad L_{2}=U_{2} \Sigma_{2} V_{2}^{\mathrm{T}}
$$

the following partitionings of $V_{1}$ and $V_{2}$ can be introduced (Golub and Van Loan, 1989):

$$
\begin{gathered}
V_{1}=\left[V_{1 r_{1}}, V_{1 n}\right], \quad V_{2}=\left[V_{2 r_{2}}, V_{2 n}\right] \\
V_{1 r_{1}}=\left[\boldsymbol{v}_{1}^{1}, \boldsymbol{v}_{2}^{1}, \ldots, \boldsymbol{v}_{r_{1}}^{1}\right], \quad V_{2 r_{2}}=\left[\boldsymbol{v}_{1}^{2}, \boldsymbol{v}_{2}^{2}, \ldots, \boldsymbol{v}_{r_{2}}^{2}\right] \\
V_{1 n}=\left[\boldsymbol{v}_{r_{1}+1}^{1}, \boldsymbol{v}_{r_{1}+2}^{1}, \ldots, \boldsymbol{v}_{n}^{1}\right] \quad V_{2 n}=\left[\boldsymbol{v}_{r_{2}+1}^{2}, \boldsymbol{v}_{r_{2}+2}^{2}, \ldots, \boldsymbol{v}_{n}^{2}\right]
\end{gathered}
$$

where the columns of $V_{1 r_{1}}=\left[\boldsymbol{v}_{1}^{1}, \boldsymbol{v}_{2}^{1}, \ldots, \boldsymbol{v}_{r_{1}}^{1}\right]$ are an orthonormal basis of the row space of $L_{1}^{r_{1}}$, indicated with $R\left(L_{1}^{r_{1}^{\mathrm{T}}}\right)$; similarly the columns of $V_{2 r_{2}}=\left[\boldsymbol{v}_{1}^{2}, \boldsymbol{v}_{2}^{2}, \ldots, \boldsymbol{v}_{r 2}^{2}\right]$ are an orthonormal basis of the row space of $L_{2}^{r_{2}}$, indicated with $R\left(L_{2}^{r_{2}^{\mathrm{T}}}\right)$. The columns of the other two matrices $V_{1 n}=\left[\boldsymbol{v}_{r_{1}+1}^{1}, \boldsymbol{v}_{r_{1}+2}^{1}, \ldots, \boldsymbol{v}_{n}^{1}\right]$ and $V_{2 n}=\left[\boldsymbol{v}_{r_{2}+1}^{2}, \boldsymbol{v}_{r_{2}+2}^{2}, \ldots, \boldsymbol{v}_{n}^{2}\right]$ are, respectively, orthonormal bases of the null spaces $N\left(L_{1}^{r_{1}}\right)$ of $L_{1}^{r_{1}}$ and $N\left(L_{2}^{r_{2}}\right)$ of $L_{2}^{r_{2}}$.

The following projectors can be then defined (Golub and Van Loan, 1989):

$$
\begin{aligned}
& P_{r_{1}}^{1}=V_{1 r_{1}} V_{1 r_{1}}^{\mathrm{T}} \quad \text { Projector onto } R\left(L_{1}^{r_{1}^{\mathrm{T}}}\right) \\
& P_{r_{2}}^{2}=V_{2 r_{2}} V_{2 r_{2}}^{\mathrm{T}} \quad \text { Projector onto } R\left(L_{2}^{r_{2}^{\mathrm{T}}}\right)
\end{aligned}
$$

Since, the MNS associated to the linear overdetermined system $\mathbf{B}=L^{r} \cdot \mathbf{p}$ lies onto the row space of $L^{r}, R\left(L_{1}^{r_{1}^{\mathrm{T}}}\right)$, contains all the MNSs obtained using the sensor array No. 1 with kernel matrix $L_{1}$ and TSVD of order $r_{1}$ and $R\left(L_{2}^{r_{2}^{\mathrm{T}}}\right)$ contains all the MNS obtained using the sensor array No. 2 with kernel matrix $L_{2}$ and TSVD of order $r_{2}$. 
According to the comparison criterion described here, matrix $V_{2 r_{2}}$ is orthogonally projected onto $R\left(L_{1}^{r_{1}^{\mathrm{T}}}\right)$. The result is a $n \times r_{2}$ matrix $P_{2 \rightarrow 1}=P_{r_{1}}^{1} \cdot V_{2 r_{2}}$, whose $j$ th column is the projection of $\boldsymbol{v}_{j}^{2}$ onto $R\left(L_{1}^{r_{1}^{\mathrm{T}}}\right)$, given by the vector $P_{r_{1}}^{1} \cdot \boldsymbol{v}_{j}^{2}$. This projection is the MNS of the linear system $\mathbf{B}=L_{r_{1}}^{1} \cdot \mathbf{p}$ when the true solution is $\boldsymbol{v}_{j}^{2}$. The norm of this projection is generally less than one because $\boldsymbol{v}_{\dot{j}}^{2} \notin R\left(L_{1}^{r_{1}^{1}}\right)$ and the higher the value of the norm the better $\boldsymbol{v}_{j}^{2}$ is catched by $L_{1}^{r_{1}}$. On the other hand, by construction, the source $\boldsymbol{v}_{j}^{2}$ is totally visible using $L_{2}^{r_{2}}$, because $\boldsymbol{v}_{j}^{2} \in R\left(L_{2}^{r_{2}^{\mathrm{T}}}\right)$ and its norm is unitary.

Since any MNS of the system $\mathbf{B}=L_{2}^{\gamma_{2}} \cdot \mathbf{p}$ can be expanded as a linear combination of the orthonormal base vectors $\left\{\boldsymbol{v}_{1}^{2}, \boldsymbol{v}_{2}^{2}, \ldots, \boldsymbol{v}_{r_{3}}^{2}\right\}$, the norms of the projected vectors $\left\{P_{r_{1}}^{1} \cdot \boldsymbol{v}_{1}^{2}, P_{r_{1}}^{1} \cdot \boldsymbol{v}_{2}^{2}, \ldots, P_{r_{1}}^{1} \cdot \boldsymbol{v}_{r_{2}}^{2}\right\}$, that are the columns of the matrix $P_{2 \rightarrow 1}$, indicate how well $L_{1}^{r_{1}}$ is capable of recovering the sources that are perfectly reconstructed by $L_{2}^{r_{2}}$. The statement above obviously holds true for vice versa, so changing 1 with 2 and defining matrix $P_{1 \rightarrow 2}=P_{r_{2}}^{2} \cdot V_{1 r_{1}}$.

The first comparison criterion can then be stated as follows.

Comparison criterion No. 1: if the columns of $P_{2 \rightarrow 1}$ have larger norms than the columns of $P_{1 \rightarrow 2}$ then sensor array No. 1 is to be preferred to sensor array No. 2 .

A second comparison criterion can be defined projecting the null space base vectors of one kernel matrix onto the row space of the other kernel matrix. More specifically, since the orthonormal vectors $\left\{\boldsymbol{v}_{r_{1}+1}^{1}, \boldsymbol{v}_{r_{1}+2}^{1}, \ldots, \boldsymbol{v}_{n}^{1}\right\}$ are a basis of the null space of $L_{1}^{\gamma_{1}}$, any linear combination of these vectors is a source that is invisible to $L_{1}^{r_{1}}$. If their projections onto $R\left(L_{2}^{r_{2}^{\mathrm{T}}}\right)$ are different from zero, they are partially visible to $L_{2}^{r_{2}}$. It is then convenient to define the matrix $Q_{1 \rightarrow 2}=P_{r_{2}}^{2} \cdot V_{1 n}$. The statement above obviously holds true for the vice versa, so changing 1 with 2 and defining the matrix $Q_{2 \rightarrow 1}=P_{r_{1}}^{1} \cdot V_{2 n}$.

The second comparison criterion can be stated as follows.

Comparison criterion No. 2: if the columns of $Q_{2 \rightarrow 1}$ have larger norms than the columns of $Q_{1 \rightarrow 2}$ then sensor array No. 1 is to be preferred to sensor array No. 2 .

\section{The biomagnetic application}

Let us consider the biomagnetic problem of Magnetocardiography described in Di Rienzo et al. (2005). We constructed a three compartment boundary element method (BEM) model out of a three-dimensional magnetic resonance image of a healthy volunteer (Figure 1). The model comprised the outer torso boundary (2,990 triangles), the left lung boundary (1,206 triangles), and the right lung boundary (1,318 triangles). We assigned homogeneous conductivities of $0.2-0.04 \mathrm{~s} / \mathrm{m}$ for the torso and the lungs, respectively. The ventricular depolarization phase of a heart beat was modelled with the help of 13 electric current dipoles, which were placed around the left ventricle. For all sources we computed the magnetic field distribution considering the sensor arrays discussed below by means of the commercial software ASA (ANT Software, Enschede, The Netherlands, www.ant-neuro.com).

For the reconstruction of the dipolar sources (solution of the IP) 13 voxels around the ventricle, each containing at the centre one dipole for each of the three orthogonal directions, were used. For the sensor setups considered below, we thus obtain an over-determined problem. By fixing the dipole locations, the inverse problem is linearised and a kernel matrix (lead field matrix) is set up. The kernel matrix contains besides the information on the geometry of the source space and the forward BEM model also the geometry of the sensor array. 
COMPEL

26,2

360

Figure 1.

Torso, lungs and ventricular blood masses in the left anterior (a) and right anterior view (b) enlarged left ventricular blood mass and position of the dipoles (c) and (d). The BEM model contains only the torso and the lungs. The ventricular blood masses are displayed only to visualize the locations of the 13 dipoles (a)

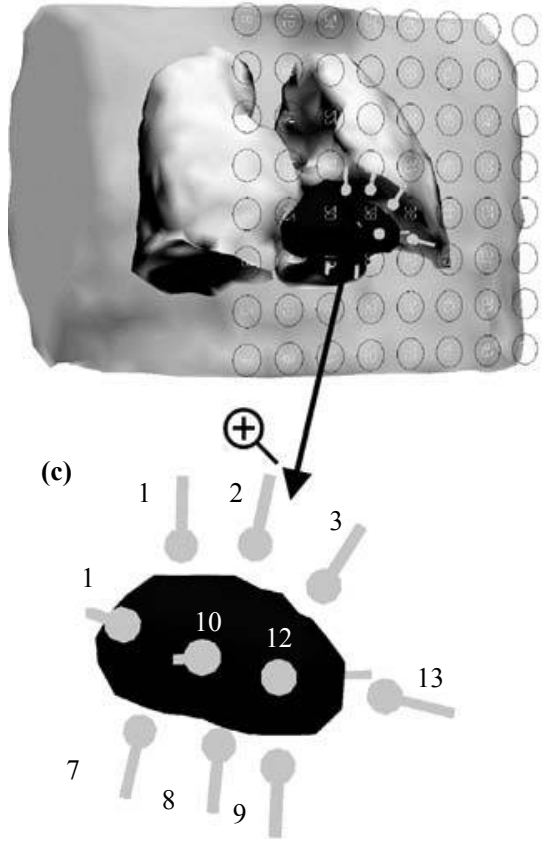

(b)

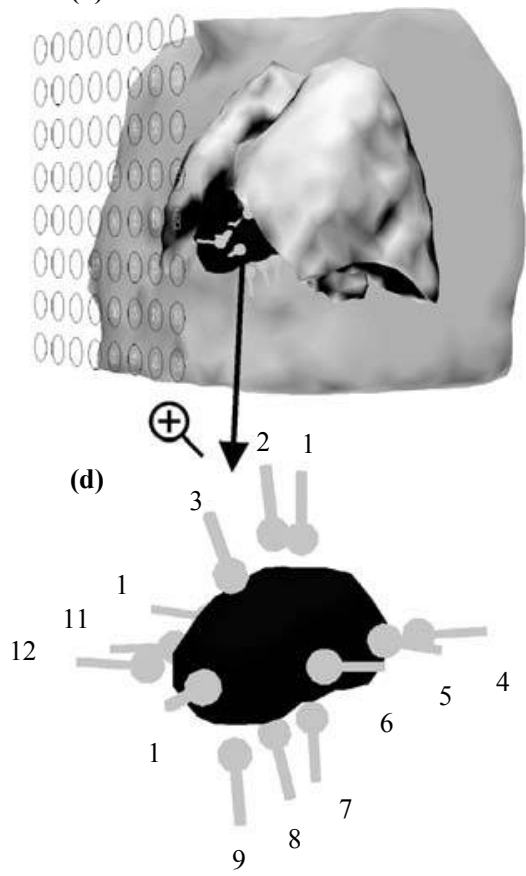

\section{A theoretical reference sensor system}

In the following section, a theoretical sensor array (gold standard system) is introduced as a gold standard reference being considered the most efficient measuring system (Figures 2 and 3). In its complete structure, it is formed by three-axial sensors lying on the faces of a box enclosing the torso: this system, of course, cannot be designed in

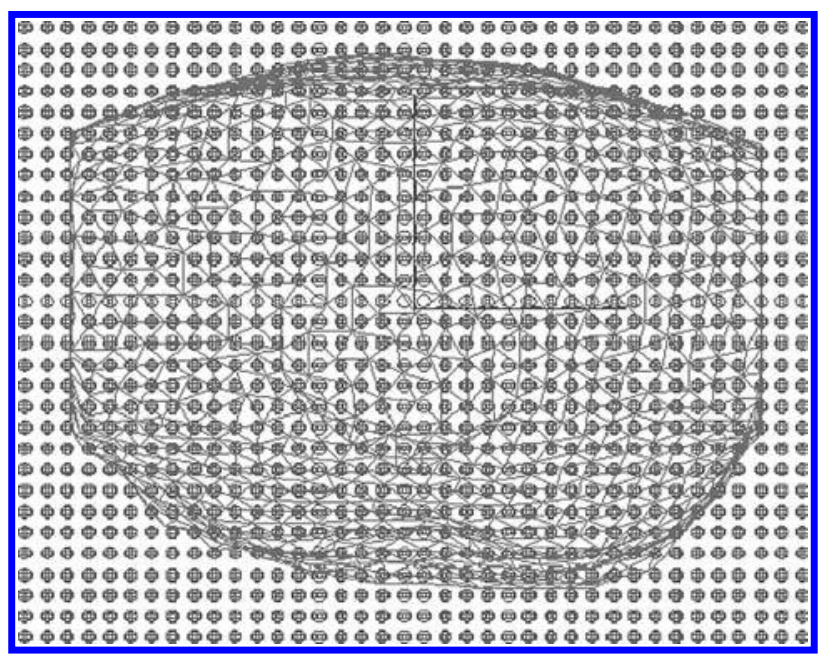

Figure 2.

Top view of the gold standard system and the BEM model of the torso 


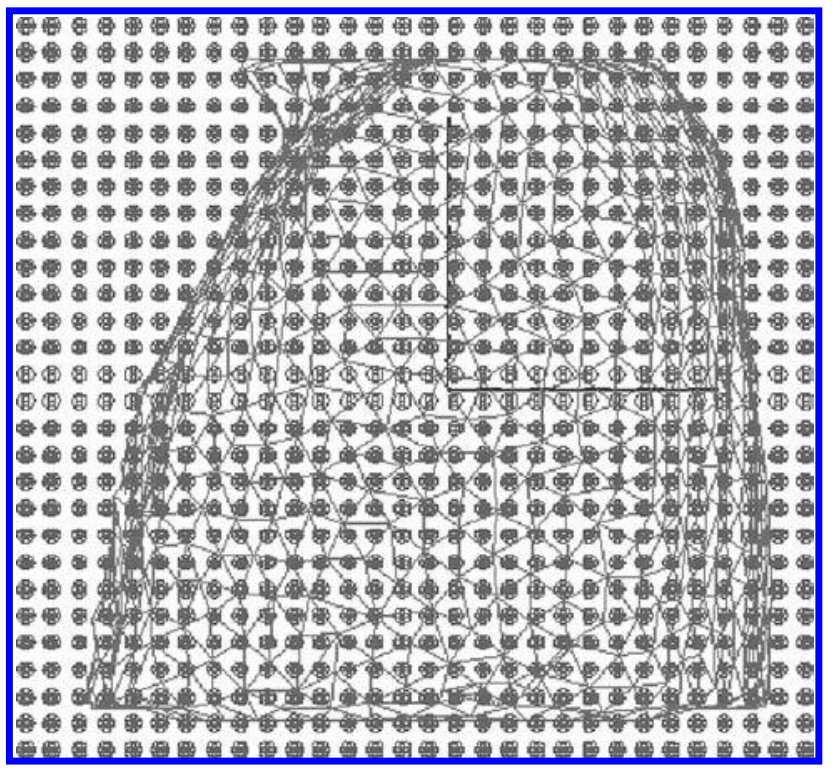

\section{Numerical comparison of sensor arrays}

361

Figure 3.

Left view of the gold standard system and the

BEM model

practice and is used here only for theoretical purposes. Magnetic sensors are located on a grid of $10 \mathrm{~mm}$ spacing. The anterior and the posterior faces, respectively, positioned in front and on the back of the thorax, consist of $28 \times 38$ three-axial sensors arrays. The right and the left sides of the system are constituted by $28 \times 30$ sensors, while the top and the bottom sides by $30 \times 38$ sensors. The total number of measuring points is $17,136(3 \times 5,712)$.

The reference system is characterized by the kernel matrix $L^{\text {gold } 3 D}$. SVD can be applied to $L^{\text {gold3 } 3 D}$ obtaining $L^{\text {gold3D }}=U^{\text {gold3D }} \Sigma^{\text {gold3D }} V^{\text {gold } 3 D^{\mathrm{T}}}$. The basic assumption is that its row space $R\left(L^{\text {gold } 3 \mathrm{D}^{\mathrm{T}}}\right)$ contains most of the reconstructable sources.

If $V^{\text {gold3 } \mathrm{D}^{\mathrm{T}}}$ is projected onto the row spaces of the two kernel matrices $R\left(L_{1}^{r_{1}^{\mathrm{T}}}\right)$ and $R\left(L_{2}^{r_{2}^{\mathrm{T}}}\right)$, it can be seen how well the complete set of reconstructable sources $R\left(L^{\text {gold } 3 \mathrm{D}^{\mathrm{T}}}\right)$ is catched by the two kernel matrices $L_{1}^{r_{1}}$ and $L_{2}^{r_{2}}$. Defining the following projectors:

$$
G_{1}=\left(V^{\text {gold3D }} V^{\text {gold3 } \mathrm{D}^{\mathrm{T}}}\right) \cdot V_{1 r_{1}}, \quad G_{2}=\left(V^{\text {gold3D }} V^{\text {gold3 } \mathrm{D}^{\mathrm{T}}}\right) \cdot V_{2 r_{2}}
$$

A third comparison criterion can be then introduced.

Comparison criterion No. 3: if the columns of $G_{1}$ have larger norms than the columns of $G_{2}$ then sensor array No. 1 is to be preferred to sensor array No. 2.

From the 3D gold standard system two other sensor arrays can be derived: a mono-axial sensor array keeping only the sensors directed normally to the sides of the box and a bi-axial sensor array measuring the tangential components of the magnetic field on the surfaces of the box.

A singular value analysis of the kernel matrices of the gold standard system measuring 1, 2 and 3 components of the magnetic field (respectively, gold1D, gold2D and gold3D arrays) is represented in Figure 4 . It can be noted how the singular values of kernel matrix $L^{\text {gold2D }}$ are approximately the same of $L^{\text {gold3D }}$, in accordance with the 


\section{COMPEL}

26,2

\section{2}

Figure 4.

Singular values normalized to their maximum value in a semi-logarithmical scale

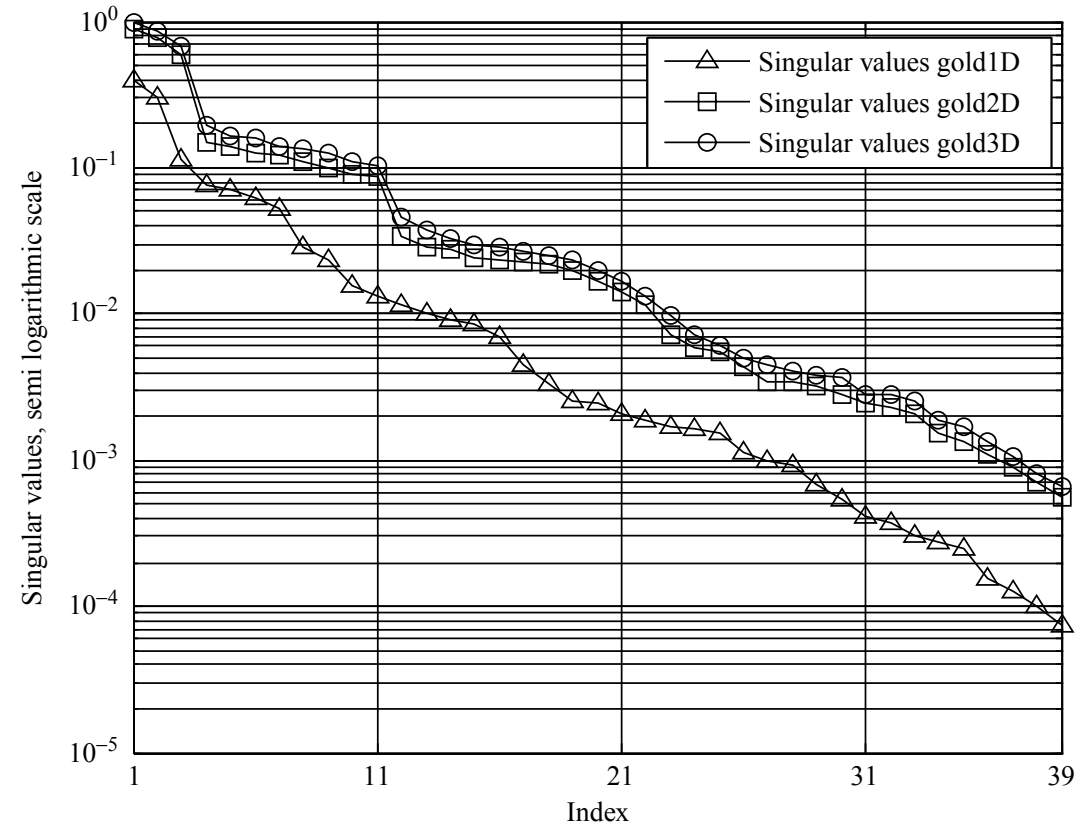

unicity theorem for the electromagnetic field, which states that the magnetic field is uniquely determined in a domain if its tangential components are known on the surface of the domain. In this case, measuring the third component of the magnetic field does not add any new information.

\section{Numerical results}

The projection method is now applied to compare the three planar sensor arrays for magnetocardiography described in Di Rienzo et al. (2005): an $8 \times 8$ array with mono-axial sensors directed normally to the plane of the array (called in the following "1D8 $\times 8$ "), an $8 \times 8$ array with three-axial sensors $(3 \mathrm{D} 8 \times 8)$ and a $14 \times 14$ array of mono-axial sensors covering the same area and also normally directed (1D14 $\times 14$ ). The latter two have approximately the same number of measurement values and the first two have the same number of measurement points. In (Di Rienzo et al., 2005) the comparison of the same sensor arrays was carried out by means of a statistical analysis, showing the superiority of the $3 \mathrm{D} 8 \times 8$ system. In this paper, the application of the projection method will lead to a consistent conclusion.

In the following, TSVD will be always applied to a kernel matrix using as threshold 1 percent of the maximum singular value, such that only the singular values higher than this threshold are kept. This choice implies different truncation numbers in TSVDs and so different dimensions of row and null spaces in the two cases under comparison.

Figures 5 and 6 show the application, respectively, of comparison criterion No. 1 and No. 2 to the arrays $1 \mathrm{D} 8 \times 8$ and $3 \mathrm{D} 8 \times 8$. The comparison shows a better performance of the 3D system. This result was foreseeable, since the 3D8 $\times 8$ system 

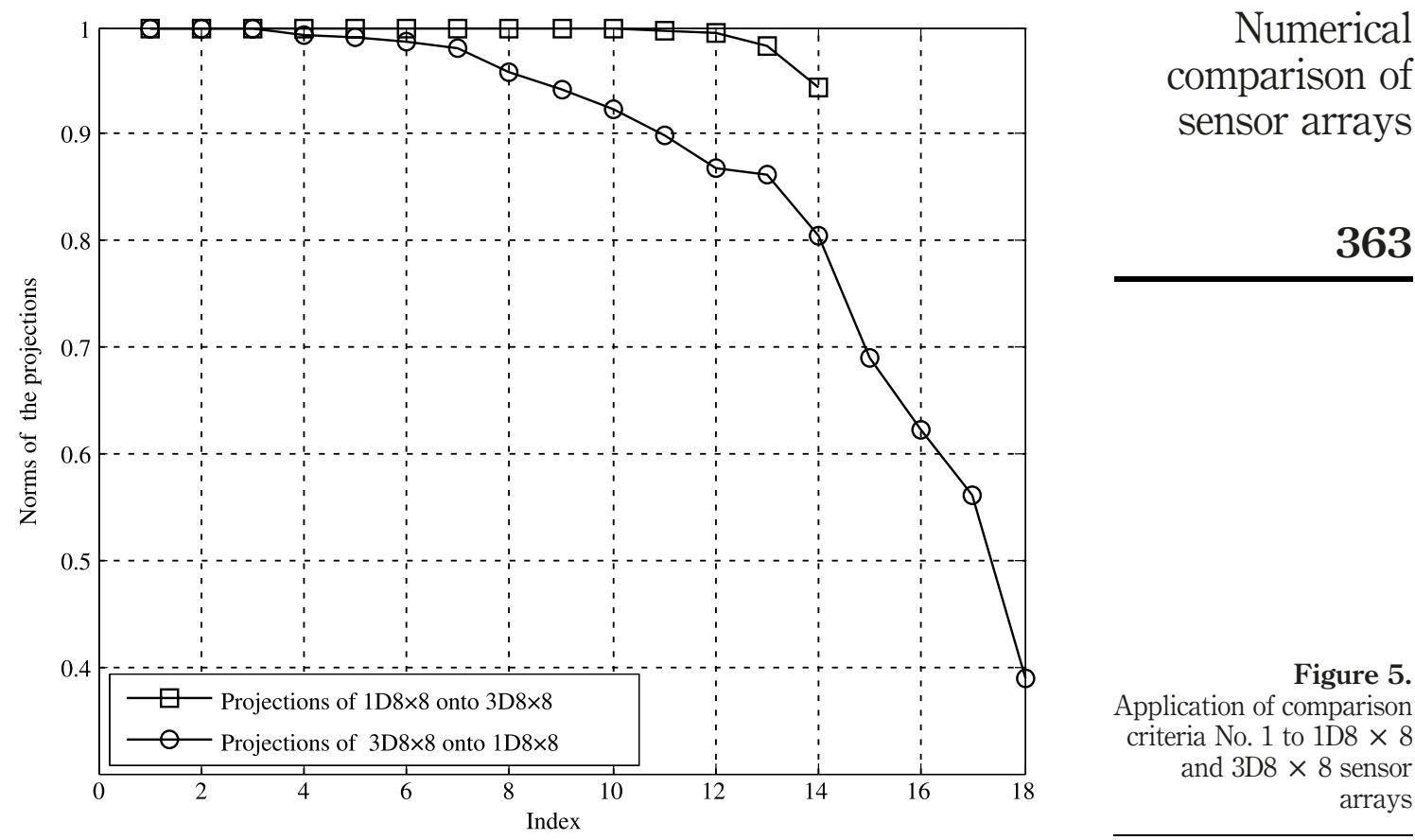

363

Figure 5.

Application of comparison criteria No. 1 to $1 \mathrm{D} 8 \times 8$ and 3D $8 \times 8$ sensor arrays

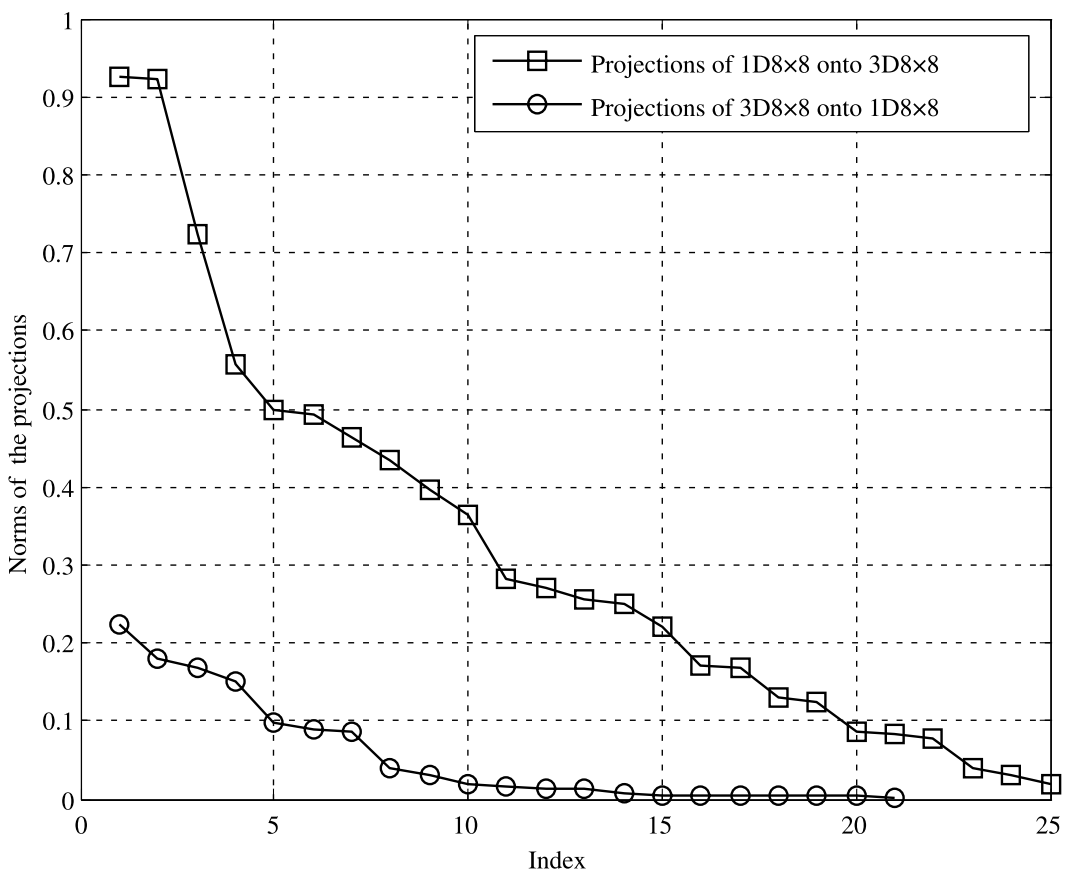

Figure 6. Application of comparison criteria No. 2 to $1 \mathrm{D} 8 \times 8$ and $3 \mathrm{D} 8 \times 8$ sensor arrays 
COMPEL

26,2

364 is characterized by three times the number of collected magnetic field data than the $1 \mathrm{D} 8 \times 8$ array.

A less expectable result characterizes the comparison between $3 \mathrm{D} 8 \times 8$ and 1 D14 $\times 14$ arrays, which still shows the superiority of the three-axial measurement system (Figures 7 and 8).

The comparison criterion No. 3 also shows the better performance of sensor array $3 \mathrm{D} 8 \times 8$ (Figure 9$)$.

\section{Conclusions}

A projection-based comparison criterion for evaluation of different sensor array geometries for linear inverse problems has been then applied to a biomagnetic problem as an applicative example, showing the superiority of three-axial sensor arrays with respect to mono-axial sensor arrays. The same results were obtained when using a five compartment BEM model (additional compartments for the ventricular blood masses) instead of a three compartment BEM model.

In our previous work (Arturi et al., 2004; Di Rienzo et al., 2005) we already considered the mono-axial and three-axial sensor arrays. However, the previous papers investigated:

- nonlinear dipole fitting (Arturi et al., 2004); and

- minimum norm solutions (Di Rienzo et al., 2005), both with the help of repeated simulations.

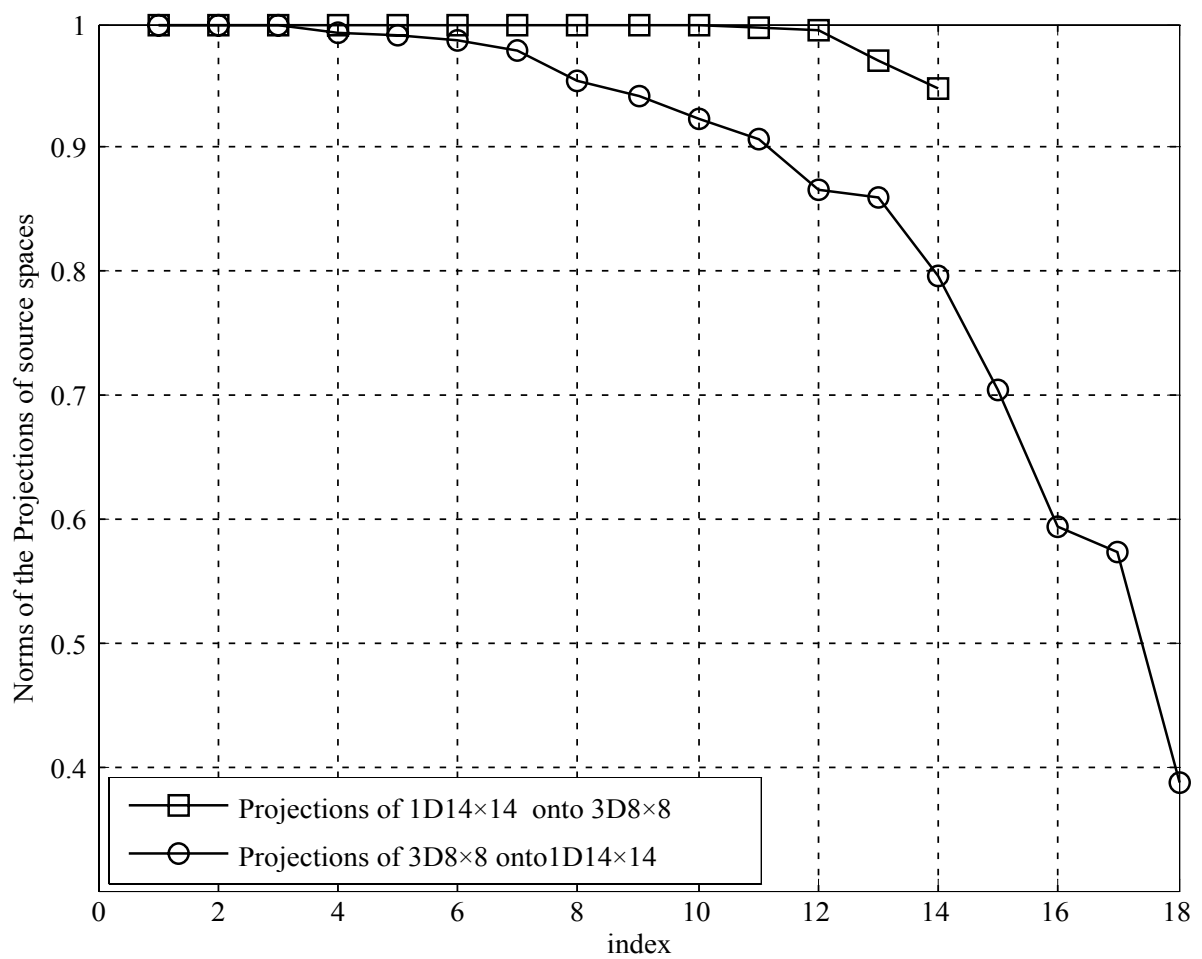

Figure 7.

Application of comparison criteria No. 1 to

$1 \mathrm{D} 14 \times 14$ and $3 \mathrm{D} 8 \times 8$ sensor arrays 

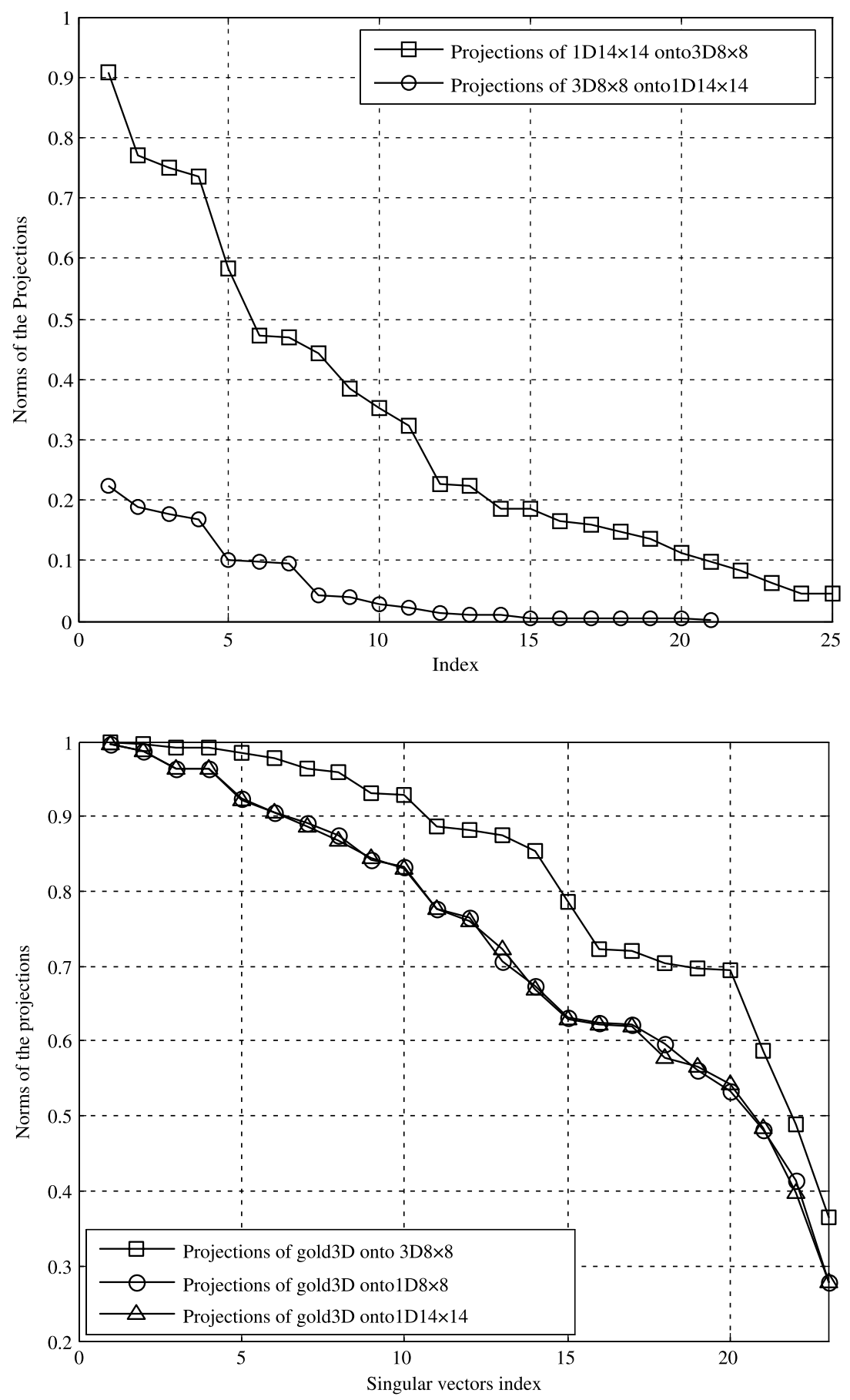

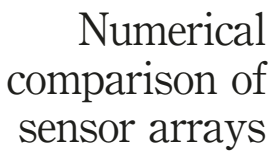

365

Figure 8. Application of comparison criteria No. 2 to $1 \mathrm{D} 14 \times 14$ and $3 \mathrm{D} 8 \times 8$ sensor arrays
Figure 9.

Application of comparison criteria No. 3 to $1 \mathrm{D} 8 \times 8$, 1 D14 $\times 14$ and 3 D $8 \times 8$ sensor arrays 
COMPEL

26,2

366
In the current paper we compare the sensor arrays with the help of a projection method in order to gain insight how one system can represent all the source configurations visible by the other system and vice versa. Furthermore, unlike the repeated simulations approach, the projection method is not influenced by the amount of noise and thus gives a more theoretical and general view on the comparison of the different sensor set-ups. Consistent over all methods we found that three-axial sensor system performs better than the mono-axial sensor systems.

The example presented here is an over-determined biomagnetic inverse problem, but the same approach would hold for other over-determined magnetostatic inverse problems. The methodology can be also applied to under-determined magnetostatic inverse problems.

For the design of biomagnetic sensor systems we conclude that three-axial systems are to be preferred.

\section{References}

Arturi, C.M., Di Rienzo, L. and Haueisen, J. (2004), "Information content in single-component versus three-component cardiomagnetic fields", IEEE Transactions on Magnetics, Vol. 40 No. 2, pp. 631-4.

Bellina, F., Bettini, P. and Trevisan, F. (2002), "Optimization analysis of the magnetic measurements on multistrand SC cables", IEEE Transactions on Applied Suberconductivity, Vol. 12 No. 1, pp. 1651-4.

Bruzzone, P., Formisano, A. and Martone, R. (2002), "Optimal magnetic probes location for current reconstruction in multistrands superconducting cables", IEEE Transactions on Magnetics, Vol. 38 No. 2, pp. 1057-60.

Chadebec, O., Coulomb, J., Cauffet, G., Bongiraud, J. and Le Thiec, P. (2002), "Recent improvements for solving inverse magnetostatic problem applied to thin shells", IEEE Transactions on Magnetics, Vol. 38 No. 2, pp. 1005-8.

Di Rienzo, L., Haueisen, J. and Arturi, C.M. (2005), "Three component magnetic field data: impact on minimum norm solutions in a biomedical application", COMPEL. The International Iournal for Computation and Mathematics in Electrical and Electronic Engineering, Vol. 24 No. 3, pp. 869-81.

Golub, G.H. and Van Loan, C.F. (1989), Matrix Computations, The John Hopkins University Press, Baltimore, MD.

Hansen, P.C. (1998), Rank-Deficient and Discrete Ill-posed Problems: Numerical Aspects of Linear Inversion, SIAM, Philadelphia, PA.

Haueisen, J., Unger, R., Beuker, T. and Bellemann, M.E. (2002), "Evaluation of inverse algorithms in the analysis of magnetic flux leakage data”, IEEE Transactions on Magnetics, Vol. 38 No. 3, pp. 1481-8.

Kemppainen, P.K. and Ilmoniemi, R.J. (1989), "Channel capacity of multichannel magnetometers", in Williamson, S.J. et al. (Eds), Advances in Biomagnetism, Plenum Press, New York, NY, pp. 635-8.

Nalbach, M. and Dössel, O. (2002), "Comparison of sensor arrangements in MCG and ECG with respect to information content", Physica C, pp. 54-258.

Rouve, L., Schmerber, L., Chadebec, O. and Foggia, A. (2006), "Optimal magnetic sensor location for spherical harmonics identification applied to radiated electrical devices", IEEE Transactions on Magnetics, Vol. 42 No. 4, pp. 1167-70. 
Scorretti, R., Takahashi, R., Nicolas, L. and Burais, N. (2004), "Optimal characterization of LF magnetic field using multipoles", COMPEL. The International Iournal for Computation and Mathematics in Electrical and Electronic Engineering, Vol. 23 No. 4, pp. 1053-61.

Shim, Y.S. and Cho, Z.H. (1981), "SVD pseudoinversion image reconstruction", IEEE Transactions on Acoustics, Speech and Signal Processing, Vol. 29 No. 4.

Vogel, C.R. (2002), Computational Methods for Inverse Problems, SIAM, Philadelphia, PA.

Ziolkowski, M., Brauer, H. and Kuilekov, M. (2006), "Identification of dominant modes in the interface between two conducting fluids", IEEE Transactions on Magnetics, Vol. 42 No. 4, pp. 1083-6.

\begin{abstract}
About the authors
Luca Di Rienzo was born in Foggia, Italy, in 1971. He received the Laurea degree and the $\mathrm{PhD}$ degree in electrical engineering from Politecnico di Milano, in 1996 in 2001, respectively. $\mathrm{He}$ is Assistant Professor with the Dipartimento di Elettrotecnica of Politecnico di Milano. His research interests are in computational electromagnetics. Luca Di Rienzo is the corresponding author and can be contacted at: luca.dirienzo@polimi.it

Jens Haueisen received a MS and a $\mathrm{PhD}$ in electrical engineering from the Technical University Ilmenau, Germany, in 1992 and 1996, respectively. Since, 2005 he is Professor of Biomedical Engineering and directs the Institute of Biomedical Engineering and Informatics at the Technical University Ilmenau, Germany. His research interests are in bioelectromagnetism. E-mail: jens.haueisen@tu-ilmenau.de
\end{abstract}

To purchase reprints of this article please e-mail: reprints@emeraldinsight.com Or visit our web site for further details: www.emeraldinsight.com/reprints 